\title{
Investigation of Coherent and Incoherent Laser Beams Propagation Through Turbulent Atmosphere
}

\author{
Sogol Tadayoni and Mahdi Shayganmanesh \\ Department of Physics, Iran University of Science and Technology, Tehran, Iran \\ Corresponding Author's Email: tadayyoni.sogol@gmail.com
}

Received: Feb. 8, 2019, Revised: Apr. 29, 2019, Accepted: Jun. 29, 2019, Available Online: Dec. 27, 2019

DOI: 10.29252/ijop.13.2.145

\begin{abstract}
In this paper propagation of combined laser beam through atmosphere is investigated. Laser beam is combined via lens array arrangement. Turbulence is considered as an affecting factor of atmosphere and its effects on transverse coherence length (Fried parameter), peak intensity, propagation efficiency, and spot size of the laser beam is investigated. Coherent and incoherent combined beam are compared. It was seen that irrespective of laser beam quality in weak and medium turbulence levels, propagation efficiency parameter in coherent and incoherent beam combining has almost the same values. Therefore replacing incoherent beam combination with coherent is proposed.
\end{abstract}

KEYWORDS: Turbulence, Incoherent beam combining, Lens array arrangement.

\section{I.INTRODUCTION}

Laser power beaming (LPB) technique can be used for kilowatt power levels and kilometer distances applications [1]. Advances in high brightness laser sources with good beam quality have enabled wide range of applications. However several physical limitations such as material heating, optical damage and nonlinear effects limit the maximum achievable power of these laser sources. In high power fiber lasers nonlinear effects such as stimulated Brillouin scattering, stimulated Raman scattering and four-waves mixing also create fundamental limitations for more power scaling [2]-[4]. Laser parameters such as spot size, peak intensity and propagation efficiency are affected in atmospheric propagation by physical phenomena include scattering as well as absorption by molecules/aerosols and atoms $[5,6]$. The factors such as rain, snow, fog, pollution, etc. affect the transmission of electromagnetic radiation, particularly optical waves through the atmosphere [7]. More than the above mentioned absorption and scattering effects, refractive index fluctuations also affect the optical wave propagation. At high power lasers, absorption has the added effect of heating the medium along the propagation path and causing to spread the beam and to noticeably reduce the peak value of the average intensity, this effect is "thermal blooming" [8]. However challenges such as laser power limitations and expensive cost of developing more powerful lasers have been led to propose the "beam combining" technique. Conventionally, there are two methods for beam combining: coherent and incoherent beam. Coherent beam combining which produces high intensities on target requires lasers with very narrow line-widths $\left(\frac{\delta \lambda}{\lambda}<10^{-6}\right)$ and good beam quality [9], [10].

In practice meet these mentioned requirements in coherent beam combining technique is more difficult than incoherent beam combining. In contrast to coherent beam combining, incoherent method does not require above mentioned conditions. Incoherent beam combining is categorized into spectral and lens array combining. In this paper a telescope is considered for focusing the beams in lens array arrangement. Fig. 1 indicates the schematic of lens array combination. According to Fig. 1, by overlapping individual 
fiber lasers beam on the target with beam director including controlled steering mirrors with adaptive optics it is possible to control the focal spot size on the target. In this research, some parameters of laser beams in propagating through atmosphere such as peak intensity, propagation efficiency, and spot size on target in both single-mode and multi-mode in incoherent beam architecture have been investigated. The propagation efficiency parameter is defined by ratio of power on target to total source laser power.

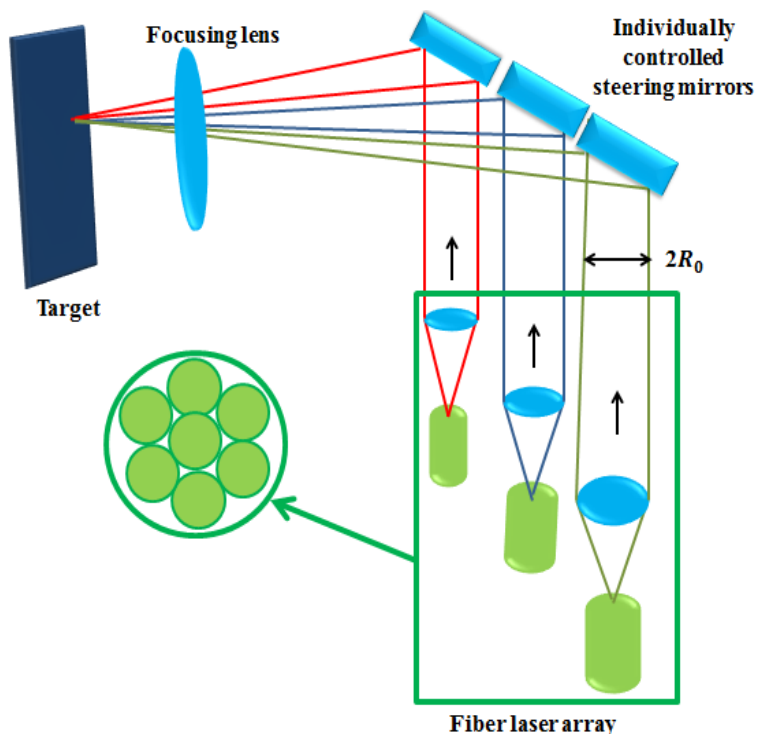

Fig. 1. Schematic diagram of lens array combination.

In 2009, Sprangle et al. investigated the propagation efficiency of incoherently combined single-mode and multi-mode fiber laser beams [11]. In this paper however, we compared the propagation efficiency of coherently and incoherently combined singlemode and multi-mode fiber lasers propagating in atmosphere with different levels of turbulence. In 2011 also, Sprangle et al. calculated the spot size of the combined beam in different turbulence levels and concluded that the spot size on target is virtually the same irrespective of used beam quality for turbulence levels of $C_{n}^{2} \geq 5 \times 10^{-15} m^{-\frac{2}{3}}$ [12]. In order to be more precise, in this paper, we have investigated other laser beam parameters such as peak intensity and propagation efficiency of incoherently combined singlemode and multi-mode lasers as a function of typical levels of atmospheric turbulence.

\section{THEORY AND ForMUlation}

\section{A. Laser Beam Propagation in Atmosphere}

The topic of laser propagation in atmosphere is a subtopic of a more general problem, the propagation of waves in turbulent medium. This general topic includes the propagation of starlight through the atmosphere, propagation of sound waves through the atmosphere and ocean, propagation of microwaves through planetary atmospheres and propagation of radio waves through the ionosphere and interplanetary space. Investigation of optical wave behavior in turbulence is important in optical communications, laser radar, imaging, adaptive optics, target designation, ranging, and remote sensing [7]. Inherent fluctuations of temperature and density of the atmosphere lead to random fluctuations in the refractive index. One of the parameters associated with turbulence is refractive index structure constant $\left(C_{n}^{2}\right)$ which characterizes the strength of turbulence [3], [7], [13], [14], [15].

As stated previously, laser beam parameters are affected by atmospheric turbulence. The long time-averaged beam radius $R(L)$ at the range $L$, is given by [11]:

$$
R^{2}(L)=\Theta_{\text {Spread }}^{2} L^{2}+R_{0}^{2}\left(1-\frac{L}{L_{\text {Focal }}}\right)^{2}
$$

In above equation $R_{0}$ is the initial spot size, $L_{\text {Focal }}$ is the focal length, $\Theta_{\text {Spread }}$ is total spreading angle and according to equation (2) its value related to diffraction $\Theta_{\text {Diff }}$, beam quality $\Theta_{\text {Quality }}$, atmospheric turbulence $\Theta_{\text {Turb }}$, mechanical jitter $\Theta_{\text {Jitter }}$ and thermal blooming $\Theta_{\text {Bloom }}$ :

$\Theta_{\text {Spread }}=\left(\Theta_{\text {Diff }}^{2}+\Theta_{\text {Quality }}^{2}+\Theta_{\text {Turb }}^{2}+\Theta_{\text {Jitter }}^{2}+\Theta_{\text {Bloom }}^{2}\right)^{\frac{1}{2}}(2)$ 
where,

$\Theta_{\text {Diff }}=M^{2} \frac{\lambda}{\pi R_{0}}$

where $M^{2}$ is the beam quality parameter and its value indicates the focusability of the laser beam, i.e. when the value of this parameter is low, therefore laser beam has good beam quality which led to less divergence and more focus on the target. In equation (2)

$$
\Theta_{\text {Quality }}=\left(M^{2}-1\right) \frac{\lambda}{\pi R_{0}}
$$

and:

$$
\Theta_{\text {Turb }}=1.6 \frac{\lambda}{\pi r_{0}}
$$

where $r_{0}$ is the transverse coherence length (Fried parameter) which is a measure of the quality of optical transmission through the atmosphere due to random inhomogeneous in the atmosphere's refractive index and is given by [10]:

$$
r_{0}=0.184\left(\frac{\lambda^{2}}{C_{n}^{2} L}\right)^{\frac{3}{5}}
$$

By ignoring thermal blooming effect for total laser power less than $\sim 100 \mathrm{~kW}$, the timeaveraged spot size $R(L)$ in equation (1) can be rewritten as:

$$
\begin{gathered}
R^{2}(L)=\left(\frac{\lambda L}{\pi R_{0}}\right)^{2}\left(M^{4}+2.9\left(\frac{R_{0}}{r_{0}}\right)^{2}\right)+ \\
\Theta_{\text {Jiter }}^{2} L^{2}+R_{0}^{2}\left(1-\frac{L}{L_{\text {Focal }}}\right)^{2}
\end{gathered}
$$

Parameters in equation (7), have been introduced previously.

Intensity of laser which is a significant parameter of beam is affected by mentioned physical phenomena and almost its quasi Gaussian distribution is preserved but its spot size spreads. The time averaged laser intensity at range $L$ is taken to have a Gaussian profile of the form:

$I(r, z=L)=I_{0} \frac{R_{0}^{2}}{R^{2}(L)} \exp \left(\frac{-2 r^{2}}{R^{2}(L)}\right)$

where $R(L)$ is the average spot size on the target which is introduced in Equation (7) and $I_{0}$ is the initial laser intensity.

\section{B. Characterizing Parameters of Intensity on Target}

The ultimate goal of beam combining is to increase the effective peak on-axis laser intensity delivered to the target. By using Equations (7) and into Equation (8), initial laser intensity and setting $L=L_{\text {Focal }}$. that gives minimum laser spot size at the particular range, the peak intensity on target at range $L$ is given by [10]:

$$
\begin{aligned}
& I_{\text {Peak }}(r=0, z=L)= \\
& \frac{6.28 P_{S}}{M^{4}+2.9\left(\frac{R_{0}}{r_{0}}\right)^{2}+\left(\frac{\pi R_{0} \Theta_{\text {Jiter }}}{\lambda}\right)^{2}}\left(\frac{R_{0}}{\lambda L}\right)^{2} \exp (-\gamma L)
\end{aligned}
$$

where $P_{S}$ is laser source power and $\gamma=\alpha+\beta$ is the atmospheric extinction coefficient that incorporates scattering and absorption physical phenomena. Value of intensity along propagation path can be affected by both the absorption coefficient $\alpha$ and scattering $\beta$ coefficient [7]. Another parameter of laser beam is propagation efficiency which its value indicates ratio of power on target to source power and is given by [11]:

$$
\begin{aligned}
\eta_{\text {prop }}= & 2 \pi P_{S}^{-1} \int_{0}^{R_{T}} d r\langle I(r, L)\rangle r= \\
& {\left[1-\exp \left(\frac{-2 R_{T}^{2}}{R^{2}(L)}\right)\right] \exp (-\gamma L) }
\end{aligned}
$$

In equation (10) radius of laser beam at the target is $R_{T}$ and $\gamma$ is extinction coefficient. In next sections of the paper, variation of peak intensity, propagation efficiency, and spot size as a function of various turbulence levels have been investigated. 


\section{Coherent and Incoherent Beam Combining}

As previously mentioned, coherent beam combining requires difficult conditions such as phase-locking and narrow line-widths. In this paper we compared incoherent and coherent beam combining to examine the possibility of replacing the incoherent method with the coherent in practical applications. In comparing both beam combining architectures, the spot size on target is calculated, in first step. Here, the spot sizes on target from Eq. (7) for coherently and incoherently combined beams with assuming $L=L_{\text {Focal }}$ are respectively given by:

$$
\begin{aligned}
& R_{C C}=\left(\frac{\lambda L}{\pi R_{B D}}\right)\left[M^{4}+2.9\left(\frac{R_{B D}}{r_{0}}\right)^{2}\right]^{\frac{1}{2}} \\
& R_{I C}=\left(\frac{\lambda L}{\pi R_{B D}}\right)\left[N M^{4}+2.9\left(\frac{R_{B D}}{r_{0}}\right)^{2}\right]^{\frac{1}{2}}
\end{aligned}
$$

where $R_{B D}$ is the radius of beam director, $M^{2} s$ are taken to be the same for both architectures and mechanical jitter $\left(\Theta_{\text {Jitter }}\right)$ has been neglected. The only difference between equations (11) and (12) is the coefficient of the beam quality terms which is related to initial spot sizes for the coherently and incoherently combined beam configurations were taken to be $R_{B D}=R_{0}$ and $R_{0} \approx \frac{R_{B D}}{\sqrt{N}}$, respectively. In Fig. 2, difference between initial spot sizes in coherent and incoherent beam combining is depicted.

According to Fig. 2(a), $N$ beams combined together and initial spot size of laser beam is considered as same as beam director size. In Fig. 2(b), several small modules $(N)$ combined together with incoherently method which one of them has initial spot size $\left(R_{0}\right)$.
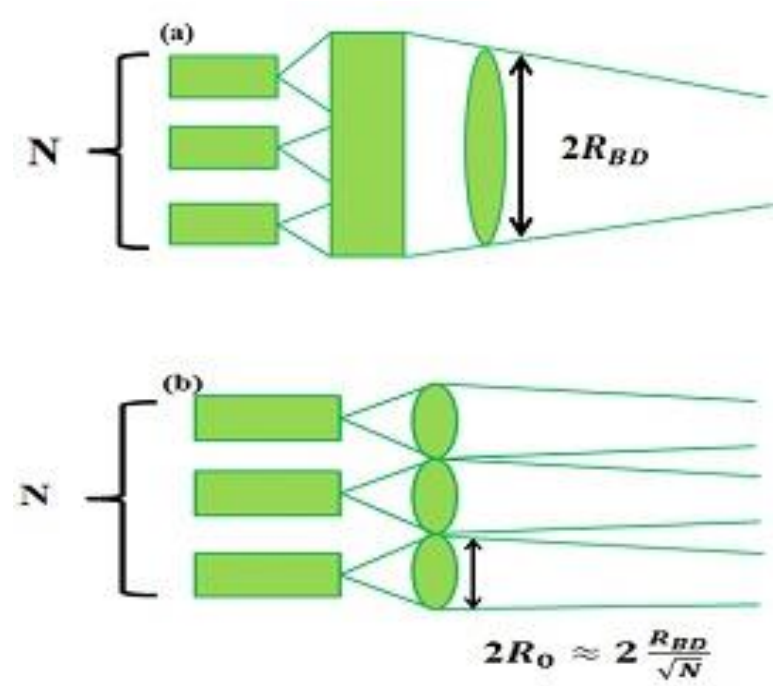

Fig. 2. Schematic of (a) coherently and (b) incoherently beam combining.

\section{III.RESULTS AND DISCUSSION}

\section{A. Effect of Atmospheric Turbulence on Transverse Coherence Length}

Turbulence affects transverse coherence length (Fried parameter) as another laser parameter. Fig. 3 is a plot of transverse coherence length versus various refractive index structure $\left(C_{n}^{2}\right)$ for three values of propagation range $(L=15 \mathrm{~km}, 10 \mathrm{~km}$, and $5 \mathrm{~km})$ where the laser wavelength is kept $\lambda=1 \mu \mathrm{m}$.

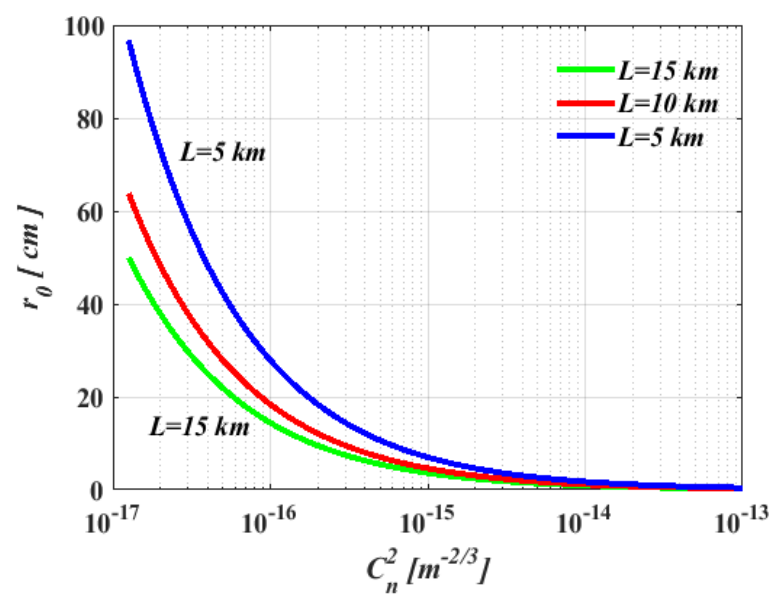

Fig. 3. Fried parameter $r_{0}$ vs. $C_{n}^{2}$ for various propagation ranges.

According to Fig. 3, the values of transverse coherence length decreases by increasing the propagation distance and refractive index fluctuations. In addition, Fig. 3 shows that in 
medium to strong turbulence $\left(C_{n}^{2}>10^{-15} m^{\frac{-2}{3}}\right)$ there is a little difference between the values of Fried parameter for various propagation distances. In the other words in strong turbulence, the effect of turbulence on the Fried parameter is independent of propagation range.

\section{B. Incoherent Single-mode and Multi-mode Beams Combining in Atmospheric Turbulence}

In incoherent beam combining arrangement, we investigated the behavior of some characterizing parameters such as peak intensity, propagation efficiency and spot size for lasers with various beam qualities in turbulent atmosphere. To illustrate, in Fig. 4, single-mode and multi-mode peak intensities on target as a function of refractive index structure constant $\left(C_{n}^{2}\right)$ for three values of beam quality $\left(M^{2}=1,3,7\right)$ are plotted. Where in all curves, wavelength is $\lambda=1 \mu \mathrm{m}$, surface area of the target $A_{T \text { arget }}=100 \mathrm{~cm}^{2}$, extinction coefficient $\gamma=0$, propagation range $L=5 \mathrm{~km}$, beam director radius $R_{B D}=30 \mathrm{~cm}$ and the number of $N=10$ fiber lasers for incoherent beam combining architecture are considered. The source power $P_{S}$ is considered to be $100 \mathrm{~kW}$.

As shown in Fig. 4, it is obvious that increasing $C_{n}^{2}$ leads to decrease in peak intensity. Increasing turbulence effect increases atmosphere fluctuations which lead to decrease in peak intensity on target. Figure 4 shows that in moderate to strong turbulence virtually the same peak intensity on target is obtained, irrespective of beam quality in incoherent beam combining architecture. Therefore in strong turbulence the effect of turbulence on laser beam is more important than beam quality parameter. It should be noted that weak turbulence results can be explained easily by the well-known diffraction theory of single and multi-mode beam propagation in vacuum. Higher order modes which are equivalent to larger $M^{2} \mathrm{~s}$ have larger divergence angle that leads to lower peak intensity of target. Regarding incoherent single-mode and multi-mode beam combining in atmospheric turbulence, Fig. 5 indicates the variation of propagation efficiency as another characterizing parameter versus turbulence intensity $\left(C_{n}^{2}\right)$ for different values of beam quality. In this example, other parameters in calculations of Fig. 5 were the same as Fig. 4.

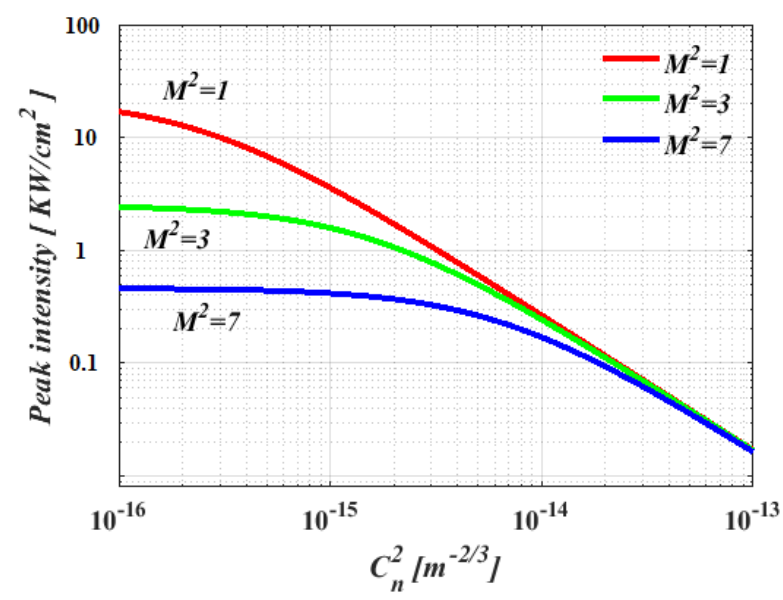

Fig. 4. Peak intensity vs. $C_{n}^{2}$ for different initial beam quality.

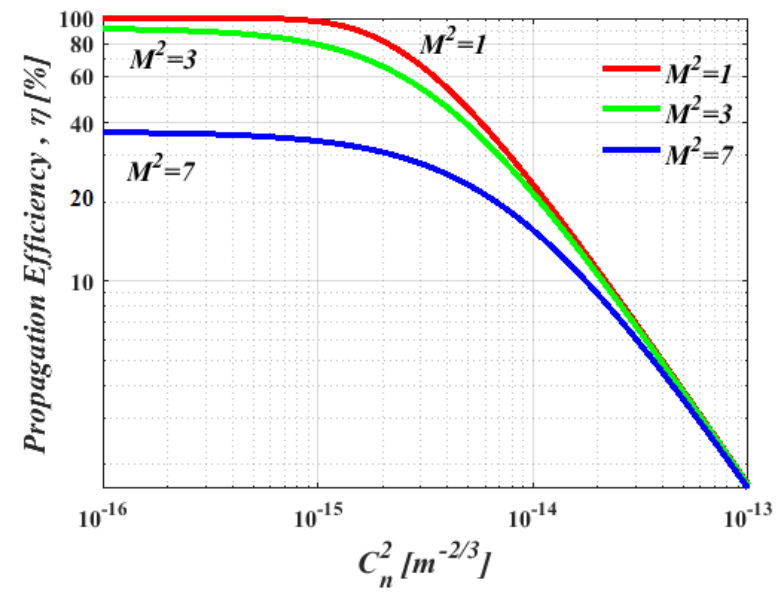

Fig. 5. Propagation efficiency vs. $C_{n}^{2}$ for different laser beam quality.

Figure 5 shows the effects of turbulence and beam quality on propagation efficiency. According to Fig. 5, it is obvious that the propagation efficiency on target is almost the same, independent of beam quality where $C_{n}^{2}>10^{-14} m^{-\frac{2}{3}}$. However in weak turbulence, 
propagation efficiency of multi-mode beam (higher $M^{2}$ ) is significantly lower than single mode which is due to divergence of the beam.

As the last parameter, in Fig. 6, spot size variation versus turbulence intensity is investigated for incoherent single-mode and multi-mode beam combining. Parameters and conditions in calculations of Fig. 6 were the same as in Figs. 4 and 5.

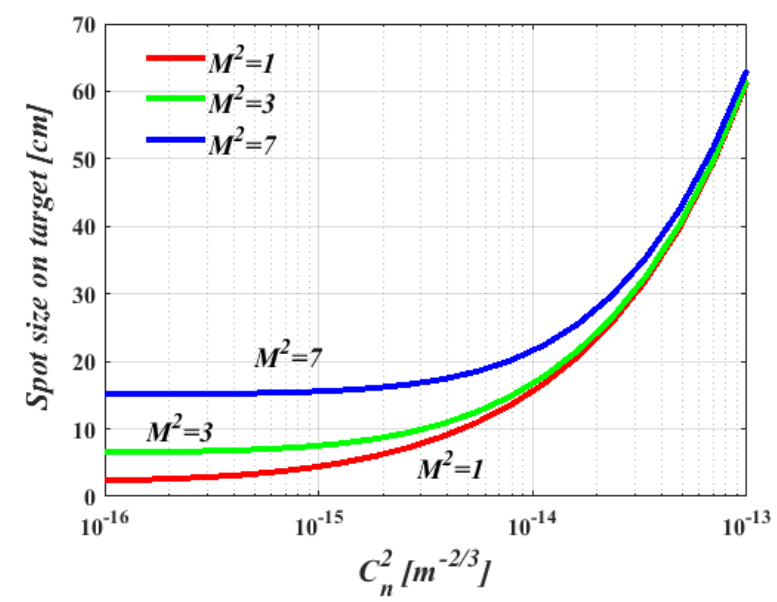

Fig. 6. Spot size vs. $C_{n}^{2}$ for different laser beam quality.

The results of Fig. 6 illustrate that in strong turbulence, spot size of the combined beam on target is almost independent of the laser beam quality.

From Figs. 4-6, it is concluded that in strong turbulent atmosphere, effect of the refractive index structure constant is more significant than the beam quality parameter.

\section{Comparison of Coherent and Incoherent Beam Combining Propagation Efficiency}

In comparing two laser beams combining architectures, the propagation efficiency on target is calculated. We kept the size of beam director $R_{B D}=30 \mathrm{~cm}$, extinction coefficient $\gamma=0$, source power $P_{S}=100 \mathrm{~kW}$, and the target is considered to be a circular disc with a surface area of $A_{T \text { arget }}=100 \mathrm{~cm}^{2}$ which is considered to be the same for both combining architectures. In these calculations, target is considered at the range of $L=10 \mathrm{~km}$ and the wavelength is considered to be $1 \mu \mathrm{m}$. In comparison, we considered $N=10$ fiber lasers for incoherent beam combining arrangement. Variations of propagation efficiency versus propagation range for both coherent and incoherent beam combining and for the three values of beam quality are shown in Fig. 7. In this Figure propagation medium is considered to be vacuum $\left(C_{n}^{2}=0\right)$. But in Figs. 8 and 9 refractive index structure constant $C_{n}^{2}$ are considered to be $C_{n}^{2}=10^{-15} m^{-\frac{2}{3}}$ and $C_{n}^{2}=10^{-13} m^{-\frac{2}{3}}$, respectively.
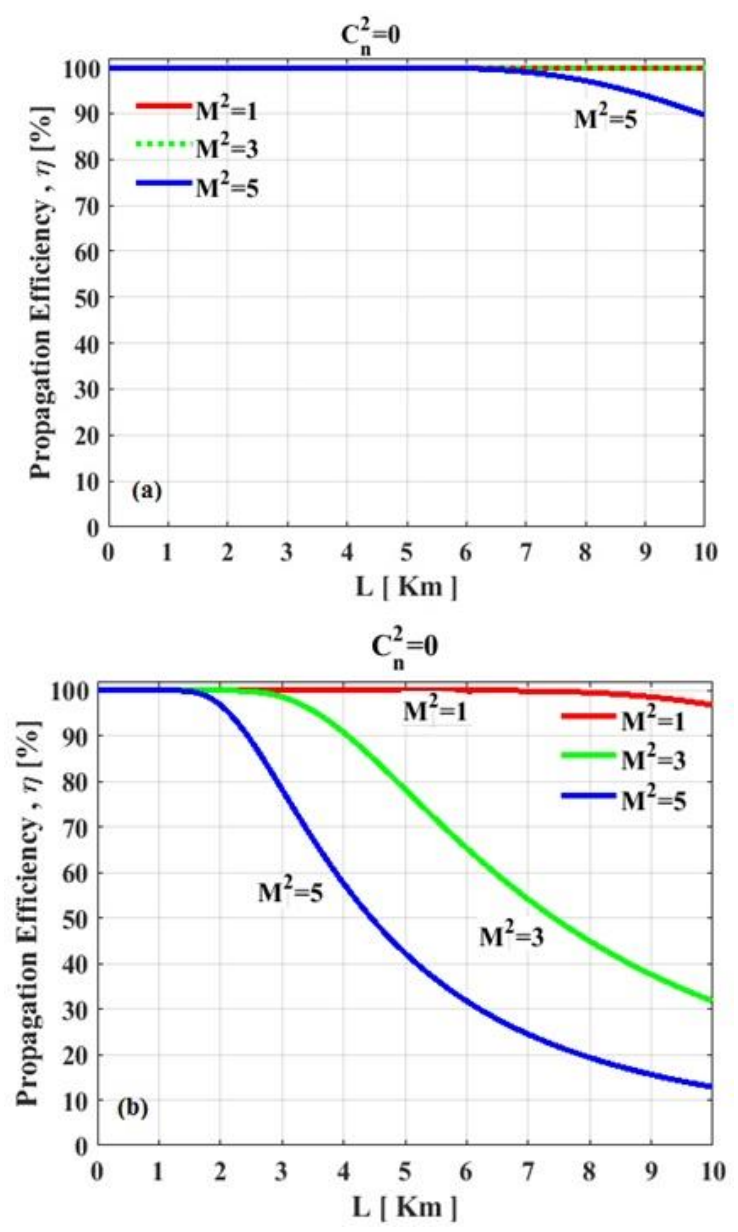

Fig. 7. Comparison of propagation efficiency versus range $(L=10 \mathrm{~km})$ for different beam quality parameters (a) coherent and (b) incoherent beam combining in vacuum $\left(C_{n}^{2}=0\right)$.

As it is seen from Fig. 7(a) for propagation of coherent combined beam in vacuum, there is very tiny difference between propagation 
efficiency of single-mode and multi-mode beams at least until $L=8 \mathrm{~km}$ ranges. This behavior can be explained by diffraction theory. Free space propagation of single and multi-mode beams in vacuum results in similar efficiencies. It should be noted that $L$ is considered to be equal to $L_{F o c a l}$.
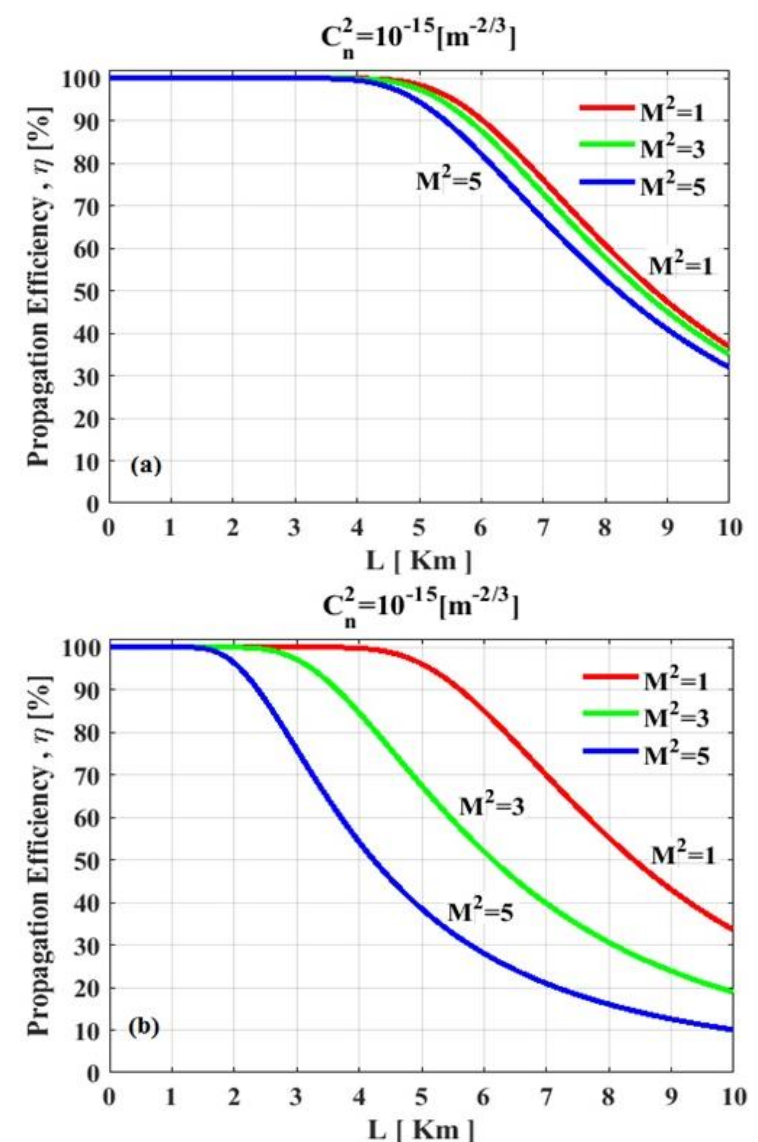

Fig. 8. Comparison of propagation efficiency versus range $(L=10 \mathrm{~km})$ for different beam quality parameters (a) coherent and (b) incoherent beam combining in strong turbulence $\left(C_{n}^{2}=10^{-13} m^{-\frac{2}{3}}\right)$.

However, Fig. 7(b) shows that for incoherent combined beam there are significant difference between single mode and multi-mode beam in long ranges of propagation. Although in this situation for short ranges lower than about $L=2 \mathrm{~km}$, propagation efficiency of single-mode and multi-mode beams are almost the same.

It is seen from Fig. 7 that for both coherent and incoherent combined beam by increasing the propagation range, difference between single-mode and multi-mode propagation efficiencies increases.

Figure 8 shows similar situation of those in Fig. 7 but for weak turbulence. As can be seen from Fig. 8(a), propagation efficiency for single mode and multi-mode beams are approximately the same for incoherent combined beam in weak turbulence, however for coherent combined there are more differences between single and multi-mode as shown in Fig. 8(b).
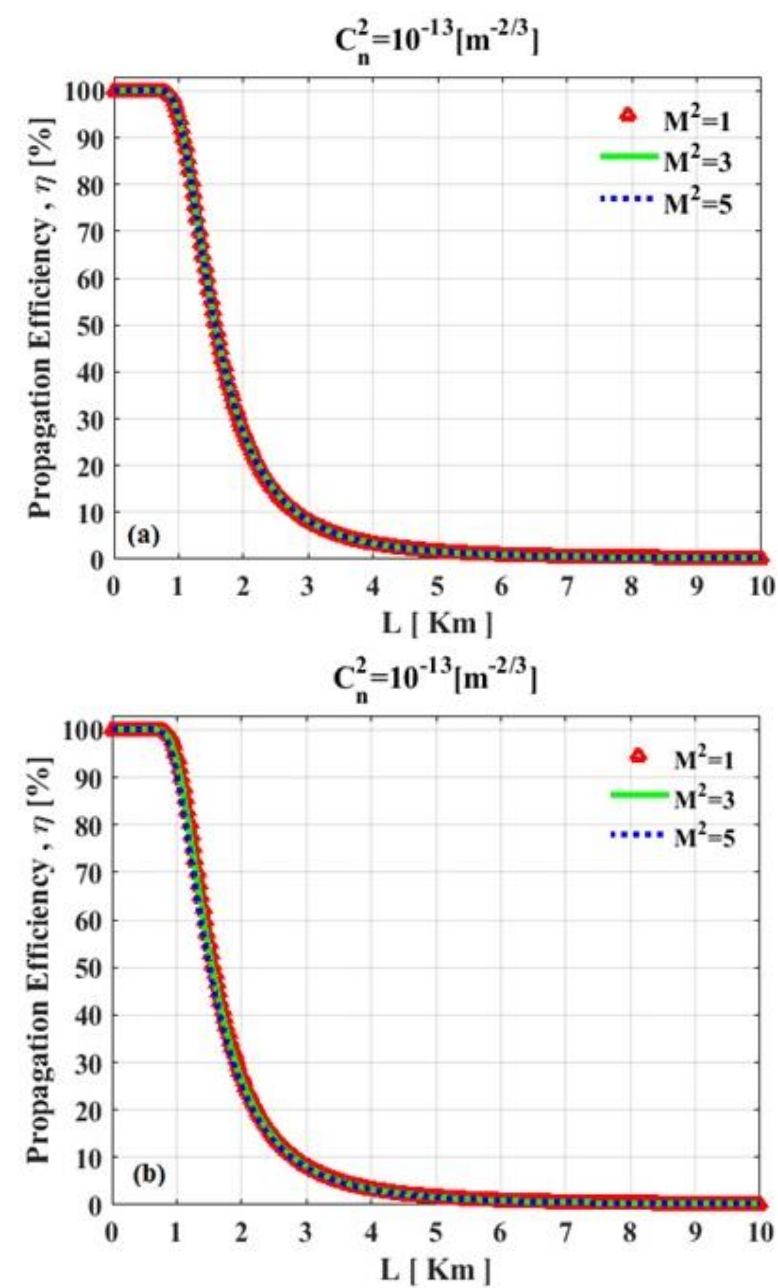

Fig. 9. Comparison of propagation efficiency versus range $(L=10 \mathrm{~km})$ for different beam quality parameters (a) coherent and (b) incoherent beam combining in strong turbulence $\left(C_{n}^{2}=10^{-13} \mathrm{~m}^{-\frac{2}{3}}\right)$.

Figure 9 surprisingly shows that there is almost no difference between single and multimode propagation efficiency in strong turbulence. Comparing Figs. 9(a) and 9(b) 
shows that even propagation efficiency of incoherent combined and coherent combined beams are the same. This recent result implies that turbulence is the most dominant effect in atmospheric propagation of combined beam.

\section{IV.CONCLUSION}

Behavior of laser parameters with various beam qualities in different turbulence levels is investigated in lens array arrangement as one of the incoherent beam combining methods. It is seen that for moderate to strong turbulence conditions, irrespective of beam quality of the used lasers in arrangement, beam parameters such as peak intensity, propagation efficiency and spot size at target is virtually the same for both coherent and incoherent combined beam. By investigation and comparing propagation efficiency of single-mode and multi-mode beams in both coherently and incoherently combining under various turbulence levels, it is concluded that in strong turbulence levels both combination methods lead to the same results.

\section{REFERENCES}

[1] T.J. Nugent and J.T. Kare, "Laser power beaming for defense and security applications," proc. of SPIE, Vol. 8045, pp. 804514 (1-8), 2011.

[2] E.P. Ippen and R.H. Stolen, "Stimulated Brillouin scattering in optical fibers," J. Appl. Phys. Lett. Vol. 539, pp. 539-541, 1972.

[3] R.G. Smith, "Optical power handling capacity of low loss optical fibers as determined by stimulated Raman and Brillouin scattering," J. Appl. Opt. Vol. 11, pp. 2489-2494, 1972.

[4] V. Roy, M. Piché, F. Babin, and G.W. Schinn, "Nonlinear wave mixing in a multilongitudinal-mode erbium-doped fiber laser," J. Opt. Express, Vol. 13, pp. 67916797, 2005.

[5] P. Sprangle, J. Peñano, and B. Hafizi, "Optimum wavelength and power for efficient laser propagation in various atmosphericenvironments," J. Directed Energy, Vol. 2, pp. 71-95, 2006.
[6] F.G. Smith, The infrared and electro-optical systems handbook, Bellingham: SPIE, Vol. 2, 1993.

[7] L.C. Andrews and R.L. Philips, Laser beam propagation through random media, SPIE press, $2^{\text {nd }}$ Ed. Bellingham, Ch. 1, 2005.

[8] M. Philbert, M. Billard, G. Fertin, and J. Lefèvre, "Thermal Blooming of high power laser beams," J. Phys. Colloques, Vol. 41, pp. C9-149, 1980.

[9] P. Sprangle, J. Peñano, B. Hafizi, and A. Ting, "Incoherent combining of high-Power Fibers Lasers for Long-range directed Energy Applications," J. Directed Energy, Vol. 2, pp. 273-284, 2007.

[10] P. Sprangle, J. Peñano, A. Ting, and R. Ficher, "High-power lasers for directed-energy applications,” J. Appl. Opt. Vol. 54, pp. F201F209, 2015.

[11] P. Sprangle, A. Ting, J. Penano, R. Ficher, and B. Hafizi, "Incoherent combining and atmospheric propagation of high-power fiber lasers for directed-energy applications," IEEE J. Quantum Electron. Vol. 45, pp. 138-148, 2009.

[12] P. Sprangle, J. Peñano, and B. Hafizi, "Beam Combining and Atmospheric Propagation of High Power Lasers," Naval Res. Lab, Washington DC Beam Physics Branch, Rep. NRL/MR/6790--11-9371(1-16), 2011.

[13] R.L. Fante, "Electromagnetic beam propagation in turbulent media," Proceedings of the IEEE, Vol. 63, pp. 1669-1692, 1975.

[14] S. Rasouli and M.T. Tavassoly, "Measurement of the refractive-index structure constant, $\mathrm{Cn} 2$, and its profile in the ground level atmosphere by moiré technique," In Proceeding of Optics in Atmospheric Propagation and Adaptive Systems IX, International Society for Optics and Photonics, Vol. 6364, pp. 63640G (1-11), 2006.

[15] S. Rasouli and M.T. Tavassoly, "Application of the moiré deflectometry on divergent laser beam to the measurement of the angle of arrival fluctuations and the refractive index structure constant in the turbulent atmosphere," J. Opt. Lett, Vol. 33, no. 9, pp. 980-982, 2008. 


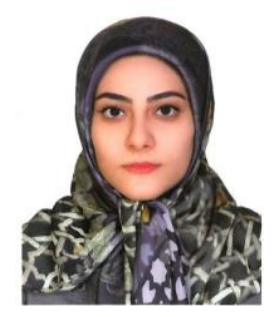

Sogol Tadayoni was born in Tehran, Iran, in 1992. She recieved her B.Sc. degree in atomic and molecular physics from Kharazmi University, Tehran, in 2015. She received her M.Sc. in atomic and molecular physics under supervision of Dr. Shayganmanesh, from Physics Department of Iran University of Science and Technology (IUST), Tehran, Iran in 2019. Her research experience is laser beam propagation through turbulent atmosphere.

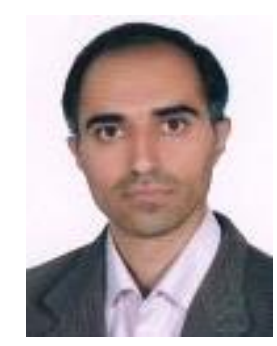

Mahdi Shayganmanesh was born in Tehran, Iran, in 1975. He received his M.Sc. degree in atomic and molecular physics from University of Tehran, Tehran, in 2001, and the Ph.D. degree in atomic and molecular physics from Iran University of Science and Technology (IUST), Tehran, in 2009. He has been a staff member of the Iranian National Center for Laser Science and Technology (INLC), Tehran, for three years. He is faculty member at the Department of Physics, IUST since 2011. His current research interests include laser development, laser beam propagation and laser material processing. 
THIS PAGE IS INTENTIONALLY LEFT BLANK. 